\title{
Amygdala response and functional connectivity during cognitive emotion regulation of aversive image sequences
}

Citation for published version (APA):

Sarkheil, P., Klasen, M., Schneider, F., Goebel, R., \& Mathiak, K. (2019). Amygdala response and functional connectivity during cognitive emotion regulation of aversive image sequences. European Archives of Psychiatry and Clinical Neuroscience, 269(7), 803-811. https://doi.org/10.1007/s00406-0180920-4

Document status and date:

Published: 01/10/2019

DOI:

10.1007/s00406-018-0920-4

Document Version:

Publisher's PDF, also known as Version of record

Document license:

Taverne

Please check the document version of this publication:

- A submitted manuscript is the version of the article upon submission and before peer-review. There can be important differences between the submitted version and the official published version of record.

People interested in the research are advised to contact the author for the final version of the publication, or visit the DOI to the publisher's website.

- The final author version and the galley proof are versions of the publication after peer review.

- The final published version features the final layout of the paper including the volume, issue and page numbers.

Link to publication

\footnotetext{
General rights rights.

- You may freely distribute the URL identifying the publication in the public portal. please follow below link for the End User Agreement:

www.umlib.nl/taverne-license

Take down policy

If you believe that this document breaches copyright please contact us at:

repository@maastrichtuniversity.nl

providing details and we will investigate your claim.
}

Copyright and moral rights for the publications made accessible in the public portal are retained by the authors and/or other copyright owners and it is a condition of accessing publications that users recognise and abide by the legal requirements associated with these

- Users may download and print one copy of any publication from the public portal for the purpose of private study or research.

- You may not further distribute the material or use it for any profit-making activity or commercial gain

If the publication is distributed under the terms of Article 25fa of the Dutch Copyright Act, indicated by the "Taverne" license above, 


\title{
Amygdala response and functional connectivity during cognitive emotion regulation of aversive image sequences
}

\author{
Pegah Sarkheil ${ }^{1,2,3} \cdot$ Martin Klasen $^{1,3} \cdot$ Frank Schneider $^{1,3} \cdot$ Rainer Goebel $^{2} \cdot$ Klaus Mathiak $^{1,3}$
}

Received: 14 February 2018 / Accepted: 25 June 2018 / Published online: 14 July 2018

c) Springer-Verlag GmbH Germany, part of Springer Nature 2018

\begin{abstract}
Emotion regulation (ER) is crucial in terms of mental health and social functioning. Attention deployment (AD) and cognitive reappraisal (CR) are both efficient cognitive ER strategies, which are based on partially dissociated neural effects. Our understanding of the neural underpinnings of ER is based on laboratory paradigms that study changes of the brain activation related to isolated emotional stimuli. To track the neural response to ER in the changing and dynamic environment of daily life, we extended the common existing paradigms by applying a sequence of emotionally provocative stimuli involving three aversive images. Eighteen participants completed an ER paradigm, in which they had to either shift their attention away from the emotionally negative images by counting backwards (AD strategy) or reinterpret the meaning of stimuli (CR strategy) to attain a down-regulation of affective responses. An increased recruitment of left-sided lateral and medial PFC was shown upon regulation of negative emotions with CR as compared to AD. Remarkably, the amygdala activation showed an increasing pattern of activation during CR. The inverse relationship between PFC and amygdala was compromised during elongated blocks of reappraisal, reflecting a reduction in engagement of the top-down prefrontal regulatory circuitry upon repeated exposure to negative stimuli. These results highlight that temporal dynamic of amygdala response and its functional connectivity differentiates AD and CR strategies in regulating emotions. Findings of the current study underscore the importance of adopting temporally variant approaches for investigating the neural effects of ER. Identifying neural systems that subserve down-regulation of negative emotions is of importance in developing treatment strategies for various forms of psychopathology.
\end{abstract}

Keywords Emotion regulation $\cdot$ Cognitive reappraisal $\cdot$ Attention deployment $\cdot$ Amygdala $\cdot$ Functional connectivity

\section{Introduction}

The ability to regulate emotions is an important part of human adaptive functioning, which is a fundamental aspect of social interaction and psychological and physical

Electronic supplementary material The online version of this article (https://doi.org/10.1007/s00406-018-0920-4) contains supplementary material, which is available to authorized users.

Pegah Sarkheil

psarkheil@ukaachen.de

1 Department of Psychiatry, Psychotherapy and Psychosomatics, Faculty of Medicin, RWTH Aachen, Pauwelsstr. 30, 52074 Aachen, Germany

2 Department of Cognitive Neuroscience, Faculty of Psychology and Neuroscience, Maastricht University, Maastricht, The Netherlands

3 JARA - Translational Brain Medicine, Aachen, Germany well-being [1]. Difficulties in emotion regulation (ER) are considered as a core mechanism underlying a variety of common symptoms of mental health problems such as mood and anxiety disorders, borderline personality disorder, substance-abuse, eating disorders and somatoform disorders [2]. Moreover, ER skills are important treatment targets for psychiatric conditions [3, 4]. Previous research has established the effectiveness of cognitive ER in reducing the experience of negative affect and physiological reaction [5-8]. Cognitive ER strategies range from attention deployment (AD), in which attention is shifted away from the stimulus, to cognitive reappraisal (CR), which is attributed to a conscious modification in the way the meaning of an emotional stimulus is interpreted [9].

Given its relevance for the well-being and mental health, a substantive body of neuroimaging studies has focused on neural systems underlying cognitive ER mechanisms in healthy [10] and clinical [11] populations. The neuroimaging 
findings have culminated in fronto-limbic models, which suggest that successful emotion regulation relies on interactions between cognitive control regions in the frontal lobe (medial, dorsolateral and ventrolateral prefrontal cortex and anterior cingulate cortex (ACC)) and emotion-responsive regions in the limbic structures of the brain such as the amygdala (for meta-analyses of these findings [12-15]). The general picture is that amygdala activation increases when confronted with affective stimuli and decreases during emotion regulation processes, mainly through the frontoamygdala down-regulating connections. This model might be oversimplified due to restrictions of the imaging protocols in measuring temporal aspects of the emotional processing. For instance, it fails to accommodate the findings about less reduction of activation in the amygdala by reappraisal than by distraction $[6,16,17]$. Furthermore, facing a series of emotional provocations is common in everyday life and questions about the extent to which the neural effects of ER unfold under ongoing emotion regulation tasks remain to be addressed. For example, what happens to brain activation in the amygdala, the key structure in processing negative emotions, during regulation of negative emotions? Can cognitive efforts during the repeated application of CR strategies result in an increase of amygdala activation?

We sought to extend the prior findings by employment of a paradigm, in which sequences of three emotional pictures were presented in each task block. Participants were required to apply either $\mathrm{CR}$ by generating cognitive reappraisal strategies or AD by distracting themselves from the emotional contents by means of backward counting. The presentation of aversive image sequences without interstimulus intervals and behavioral response collection was used to elicit a continued emotional response. The paradigm was restricted to the regulation of negative emotions, given the relevance of negative affects to psychopathological disorders and the potential that findings will translate to clinical intervention. By assessing the fMRI responses to series of emotional stimuli, we aimed at investigating nonlinear and time-sensitive amygdala activation as a function of the emotion regulation strategy. This study relied on regulation of a series takes a critical step in understanding the neural basis of ER in changing environments. Such knowledge may ultimately lead to new training protocols to further improve the emotion regulation skills in treatment settings.

We assumed that the chronological properties of amygdala responses and the amygdala-centered connectivity can give insight into the dynamics underlying the differential mechanisms of emotion regulation. The central hypothesis of our paradigm was that the manipulations of the ER strategies upon the presentation of a sequence of negative stimuli reveals distinctions regarding temporal dynamics of amygdala activation and the functional coupling between amygdala and frontal regions.

\section{Materials and methods}

\section{Subjects}

Eighteen right-handed volunteers [mean age $=24.33$ years (SD 3.36); mean education: 16.72 years (SD 1.67); 10 females] were recruited through advertisement at the Maastricht University. The participants did not have any history of neurological or psychiatric conditions and all gave informed consent. The study protocol was approved by the Ethics Committee of the Faculty of Psychology, Maastricht University.

\section{Stimuli}

Hundred-sixty pictures with negative valence were selected from the International Affective Picture System (IAPS). The IAPS comprises pictures with validated valence $(1-9 ; 1=$ extremely negative) and arousal (1-9; $1=$ no arousal) [18]. The averaged valence of the selected image set was $2.7(\mathrm{SD}= \pm 0.6)$ with the average arousal of $5.6(\mathrm{SD}= \pm 0.8)$. A pilot test, in preparation for the major study, confirmed the adequacy of 24 ER blocks (divided in $\mathrm{CR}$ or $\mathrm{AD}$, each consisting of 3 stimuli) to elicit robust fMRI responses. 24 images were randomly selected for each participant. Half of the individual stimuli were assigned to either the AD or the CR condition. Three repetitions of each stimulus were presented during the whole presentation. The order of repetitions was semi-randomized, so that images were not repeated within a trial. Based on subjects' feedback gathered in the pilot test, this design was adopted to reduce the number of novel images and the stress imposed on the subjects by the sight of highly negative images.

\section{Training}

Before MR scanning, participants underwent a training of cognitive appraisal for approximately $45 \mathrm{~min}$. They were taught to generate interpretations of the images that would help to reduce negative emotions (for instance, by generating thoughts about an improvement in the future, assuming the perspective of a professional, or focusing on technical aspects). No training was offered for attention deployment. Subsequently, subjects carried out a practice test including twelve trials (with a sample of pictures different from ones used during scanning), which familiarized them with the experimental procedure. 


\section{Task}

The task during scanning session consisted of 12 "AD" and 12 "CR" blocks, in which three consecutive pictures were presented. Subjects were instructed to first watch each picture and experience the emotional content. After $1000 \mathrm{~ms}$, an instruction cue appeared in the middle of the picture before participants either count backwards (AD condition cued with ' $X$ ') or applied the cognitive reappraisal strategies (CR condition cued with ' + '). Each picture was shown for $4500 \mathrm{~ms}$ and each block comprised 3 pictures. Each block was followed by an interval with a blank display lasting $9000 \mathrm{~ms}$. The order of AD and CR blocks were randomized. The experimental session was interleaved by 5 randomly positioned baseline blocks (grey display for $21 \mathrm{~s}$, Fig. 1).

\section{fMRI scanning}

Participants were scanned using a 3 T Siemens Magnetom Allegra head-scanner (Siemens Medical Systems, Erlangen, Germany) to acquire echo planar $\mathrm{T} 2 *$-weighted images (EPI) with blood oxygenation level dependent (BOLD) contrast [time to echo $(\mathrm{TE})=30 \mathrm{~ms}$, flip angle $=90^{\circ}$, matrix size $=64 \times 64]$. With a repetition time of $\mathrm{TR}=1500 \mathrm{~ms}$, 441 volumes were acquired in a single continuous run ( $11 \mathrm{~min})$. Each volume with 26 axial slices and 3-mm in-plane resolution covered the entire brain except for cerebellum (see supplementary material for the averaged

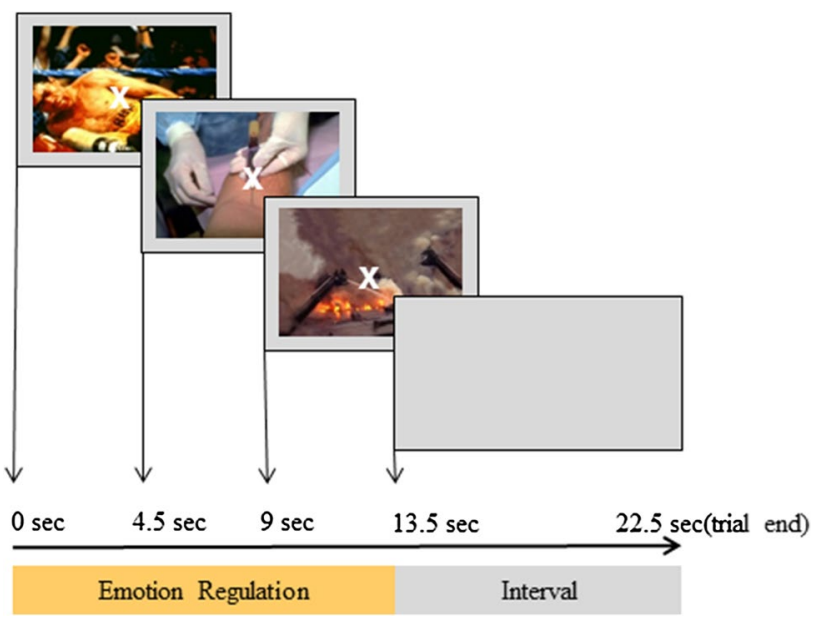

Fig. 1 Experimental paradigm. Participants were presented with a sequence of three aversive images (each $4.5 \mathrm{~s}$ ) followed by an Interval $(9 \mathrm{~s})$. They were instructed to observe each picture and apply AD or CR strategies to regulate the negative emotions. The order of the ER strategies was randomized and was based on a visual cue, which appeared in the middle of the picture. " $X$ " cued AD (like illustrated in the picture) and "+" cued CR. $A D$ attention depletion, $C R$ cognitive reappraisal image from all participants). To allow for $\mathrm{T} 1$ equilibration effect the first three volumes of each run were discarded. After functional imaging a T1-weighted structural image (TE=9 ms, TI $900 \mathrm{~ms}$, TR $1900 \mathrm{~ms}, 1 \mathrm{~mm}$-isotropic 3d with $256 \times 256 \times 276$ matrix) was acquired.

\section{fMRI data analysis: whole-brain and region of interest (ROI) analysis}

This analysis was undertaken using BrainVoyager QX 2.3 (Brain Innovation, Maastricht, The Netherlands). Preprocessing of imaging data included following steps: slice scan time correction, 3D motion detection and correction with rigid body transformations, inhomogeneity correction and segmentation of anatomical images, functional-anatomical coregistration, transformation to Talairach space and spatial smoothing with a Gaussian kernel of FWHM 6 mm.

Whole-brain analysis across all participants was conducted based on General Linear Model (GLM) to contrast $\mathrm{AD}$ and $\mathrm{CR}$ emotion regulation strategies. Statistical analyses were first carried out using a GLM at the single subject level. Parameter estimates were calculated for each voxel based on design matrices which included predictors for $\mathrm{AD}$ and $\mathrm{CR}$ relative to the baseline.

On the first-level, modelling considered the three presented images as one block of the duration of $13.5 \mathrm{~s}$ and each block with three images was modelled as single predictor. Then, each subject's parameter estimates entered separately the multiple subject GLM calculation (random effects model) and were used to contrast the two emotion regulation strategies. Statistical group maps were generated to represent significant results at the $p<0.05$ level, corrected for multiple comparisons with false discovery rate (FDR) approach.

BOLD response time course analysis was performed by extracting the timecourses and event-related averaging from bilateral amygdala ROI after running a random effects GLM, in which the predictors were convolved with "Two gamma HRF", a hemodynamic response function (HRF). The localization of amygdala was based on SPM Anatomy toolbox version 1.5 [19]. Laterobasal amygdala was selected, which is known to be the main part of amygdala with a role in integrating environmental information through connections to sensory cortices and prefrontal lobe [20]. Regression parameters for linear increase were compared with a paired $t$ test.

\section{fMRI data analysis: connectivity analysis}

The connectivity analysis examined how amygdala time course changed in relation to other brain voxels. This examination was performed using the CONN toolbox, version 16.b (http://www.nitrc.org/projects/conn, RRID:SCR_009550) [21], which includes group-level seed-based connectivity analysis. The preprocessing steps 
for functional (realignment \& unwrap, slice-timing correction, normalization, ART-based outlier detection, and smoothing) and structural (segmentation and normalization) scans. Settings for the preprocessing were chosen in analogy to the above-mentioned preprocessing steps using BrainVoyager. Confounding effects (WM, CSF, and realignment parameters), as identified by CONN, were entered as confounds in a first-level analysis.

Correlation coefficients were calculated for each subject by extracting the BOLD time course from amygdala as the seed region of interest and then by computing the correlation coefficient between that and the time courses from all other brain voxels during each condition. Correlation maps were produced based on this computation. Statistical comparisons were applied to the main conditions CR and AD. Based on the results of the ROI-analysis EARLY and LATE phases of CR blocks were defined as conditions in post hoc fashion. The presentation time of the first and the third pictures (each $4.5 \mathrm{~s}$ ) outlined EARLY CR and LATE CR conditions respectively. Overall, the correlations were compared between conditions (CR vs. AD; LATE CR vs. EARLY CR) for all participants by $t$ tests run in CONN (second level analysis) and positive/negative functional correlations with seed areas explored in SPM8. Significant differences in Amygdala FC between (1) CR and AD condition as well as (2) EARLY CR and LATE CR phase were determined using family-wise error (FWE) whole-brain-corrected threshold of $p<0.05$.

\section{Results}

\section{Whole-brain analysis}

The contrast $[\mathrm{CR}>\mathrm{AD}]$ in the GLM revealed an activation network including bilateral and left-dominant medial and lateral PFC, bilateral temporal lobe structures, posteriorcingulated cortex, left thalamus, left caudate nucleus, left fusiform gyrus, and extrastriate cortex in left occipital lobe (see hot colors in Fig. 2). Bilateral inferior parietal lobule (IPL) activation was higher in [AD $>C R]$ GLM contrast (see cold colors in Fig. 2).

\section{ROI-based analysis}

Event-related averaged BOLD response in bilateral amygdala showed a delayed enhancement during the CR blocks (Fig. 3). Paired-sample $t$ Test revealed that the increase of amygdala responses was significant higher in the CR as compared to the AD condition.

During the CR condition, amygdala activity increased over time [slope $>0.070 \pm-0.083 ; t(17)=3.60 ; p<0.005$ ] in contrast to the $\mathrm{AD}$ condition [slope $>0.025 \pm-0.076$; $t(17)=1.42 ;$ n.s.]. One-sided two-sample $t$ test confirmed that the $\mathrm{CR}$ slopes were significant larger than $\mathrm{AD}$ slopes $[t(17)=2.03, p<0.05]$.

Further exploration of the differences between $\mathrm{AD}$ and $\mathrm{CR}$ during individual pictures revealed that amygdala activation during ER of the third picture was larger than in the $\mathrm{AD}$ condition $[t(17)=4.01, p<0.005]$.

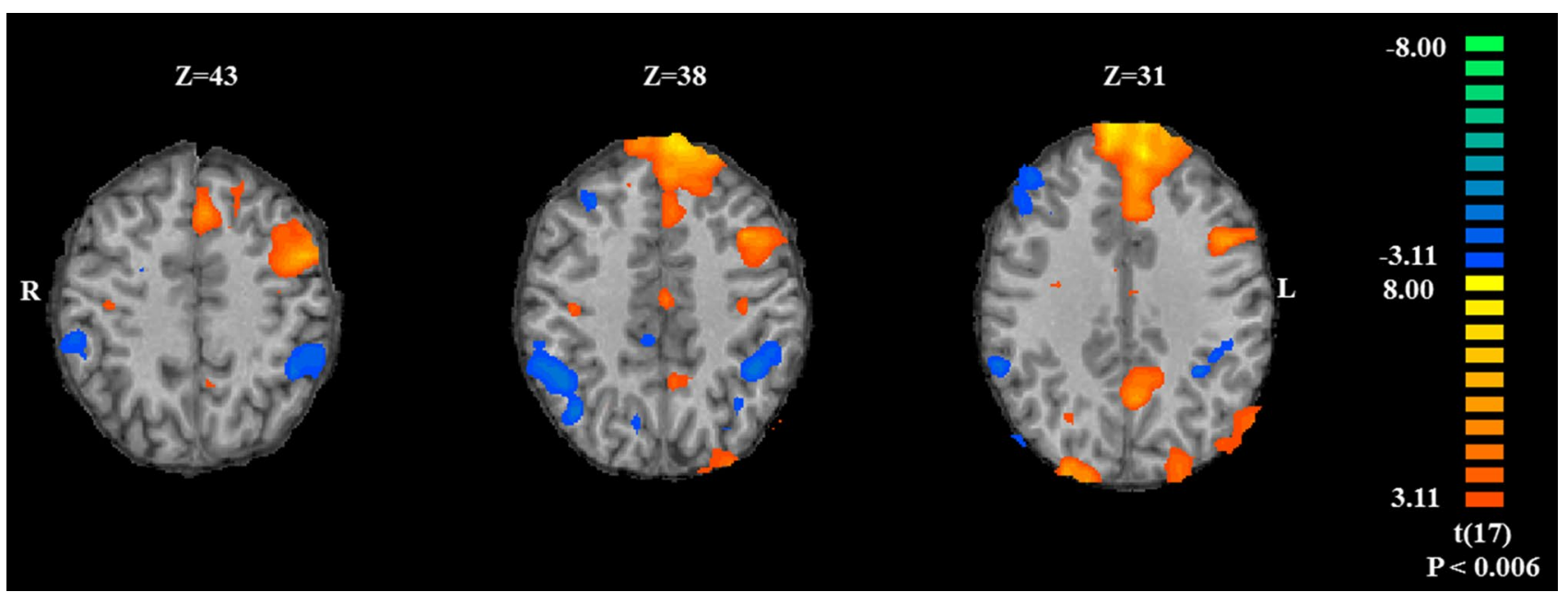

Fig. 2 GLM random-effect group maps for the [CR $>$ AD] revealed an activation network including bilateral and left-dominated medial PFC, left lateral PFC, bilateral middle temporal gyrus, posterior cin- gulated cortex, left thalamus, left fusiform gyrus, and extrastriate cortex in bilateral occipital lobe. [AD $>C R]$ resulted in bilateral inferior parietal lobule and right dorsolateral PFC activations 


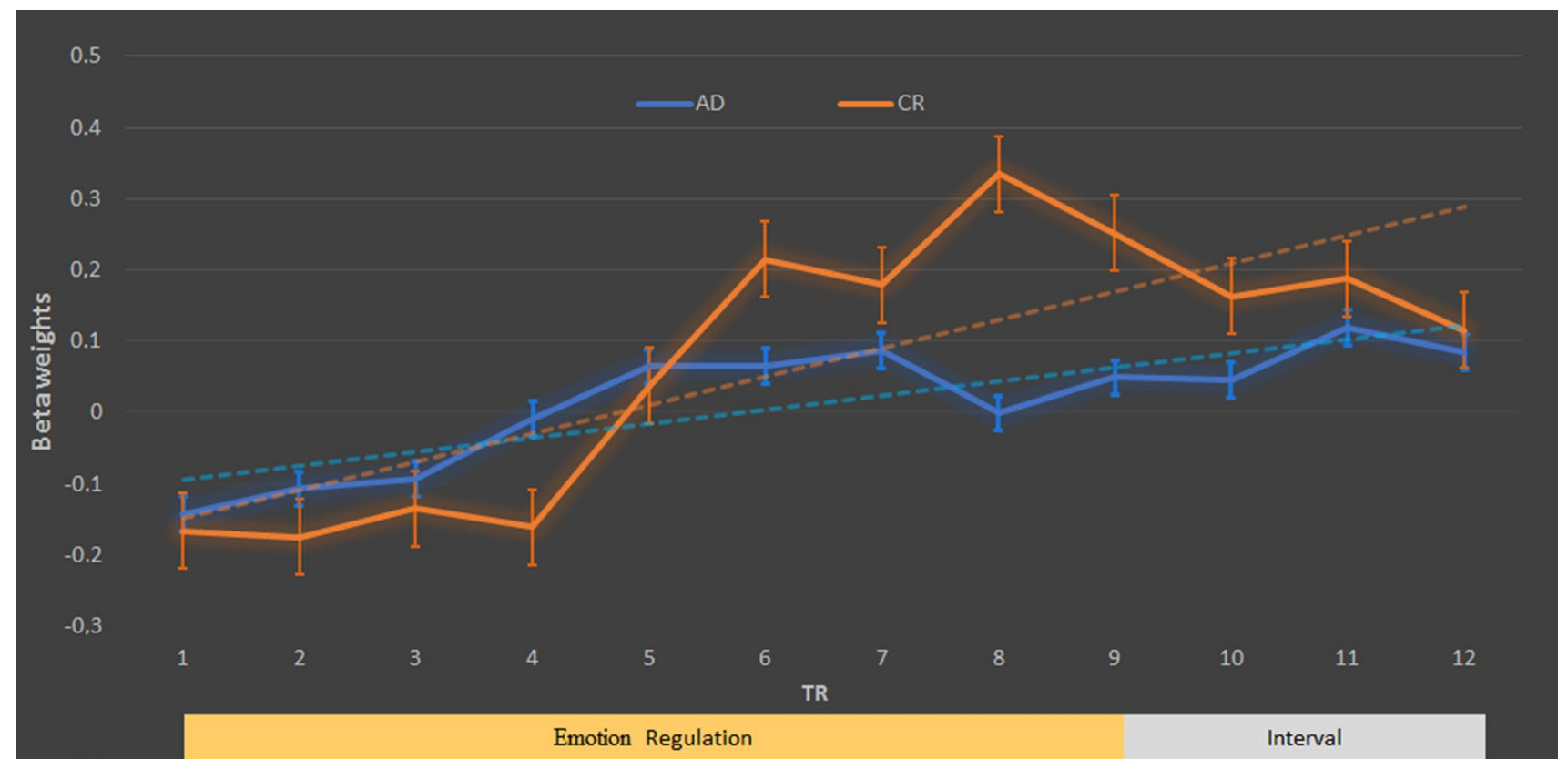

Fig. 3 Group-averaged timecourse of amygdala activation during AD and CR blocks. $A D$ attention depletion, $C R$ cognitive reappraisal. $X$ : point of image acquisition (TR), $Y$ : beta weights. The dotted lines indicate the slope of time course curves. The error bars indicate the standard errors
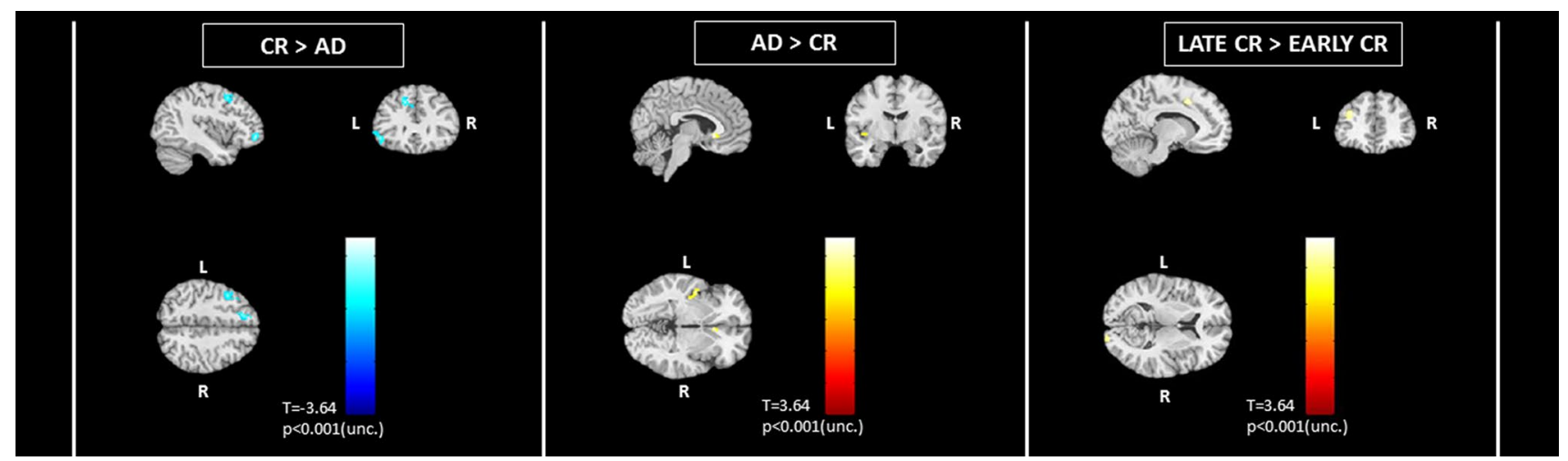

Fig. 4 Functional connectivity contrast maps-based correlation coefficient between time course of amygdala activation and the time course from all other brain voxels for $\mathrm{CR}>\mathrm{AD}$ (left), $\mathrm{AD}>\mathrm{CR}$ (middle),

\section{Connectivity analysis}

During CR compared to AD, significant negative correlations $[p$ (FDR-corrected, cluster-level) $<0.001]$ emerged between amygdala and cortical regions within left PFC (Table 1a, Fig. 4), which suggest down-regulating connections of these regions with amygdala. Positive correlations were found between amygdala and the insula and subgenual ACC [ $p$ (FDR-corrected, cluster-level) < 0.05; Table 1a, Fig. 4].

During cognitive reappraisal comparing the LATE to the EARLY phase (LATE CR > EARLY CR), we observed and LATE CR $>$ EARLY CR (right). $A D$ attention depletion, $C R$ cognitive reappraisal

a less negative correlation between the amygdala and the VLPFC, anterior ACC and visual cortex [ $p$ (FDR-corrected, cluster-level) $<0.05$ ], indicating a lower downregulation of amygdala by these cortical regions during later appraisal (Table 1b, Fig. 4 ). Frontal medial cortex, right-hemispheric temporal lobe, and precuneus exhibited higher negative correlations with amygdala during the LATE reappraisal phase (Table 1b, Fig. 4c). 
Table 1 Clusters (MNI coordinates of the peak voxel) showing a significant functional connectivity with amygdala

\begin{tabular}{|c|c|c|c|c|}
\hline & Region & Clusters $(x, y, z)$ & $\begin{array}{l}\text { Size }(\mathrm{Nr} \text { of } \\
\text { voxels })\end{array}$ & $\begin{array}{l}\text { Cluster-level } p \\
\text { FWE-corrected }\end{array}$ \\
\hline \multicolumn{5}{|l|}{ (a) $\mathrm{CR}>\mathrm{AD}$} \\
\hline \multirow[t]{2}{*}{ Positive correlations } & Left insula & $-48-04-04$ & 48 & 0.016 \\
\hline & Right sgACC & $+04+20-06$ & 47 & 0.016 \\
\hline \multirow[t]{5}{*}{ Negative correlations } & Left $\mathrm{SFG}^{\mathrm{a}}$ & $-18+50+40$ & 325 & 0.001 \\
\hline & Left frontal pole ${ }^{\mathrm{b}}$ & $-38+48-08$ & 288 & 0.001 \\
\hline & Left $\mathrm{IFG}^{\mathrm{c}}$ & $-48+28-08$ & 238 & 0.001 \\
\hline & Left frontal pole ${ }^{b}$ & $-20+58+24$ & 178 & 0.001 \\
\hline & Left precentral gyrus & $-44+10+48$ & 165 & 0.001 \\
\hline \multicolumn{5}{|c|}{ (b) LATE CR > EARLY CR } \\
\hline \multirow[t]{3}{*}{ Positive correlations } & Left frontal pole ${ }^{\mathrm{b}}$ & $-34+42+26$ & 107 & 0.001 \\
\hline & Left anterior ACC & $-12+10+42$ & 50 & 0.026 \\
\hline & Right occipital pole ${ }^{\mathrm{d}}$ & $+18-96+08$ & 48 & 0.026 \\
\hline \multirow[t]{3}{*}{ Negative correlations } & Frontal medial lobe & $+04+36-16$ & 40 & 0.038 \\
\hline & Right temporal pole & $+50+18-30$ & 40 & 0.038 \\
\hline & Right precuneus & $+26-54+10$ & 34 & 0.048 \\
\hline
\end{tabular}

$A C C$ anterior cingulate cortex, $S g A C C$ subgenual ACC, $S F G$ superior frontal gyrus, $I F G$ inferior frontal gyrus

${ }^{\mathrm{a}}$ Corresponds to DLPFC

${ }^{\mathrm{b}}$ Corresponds to VLPFC

${ }^{\mathrm{c}}$ Corresponds to orbitofrontal cortex

${ }^{\mathrm{d}}$ Corresponds to visual cortex

\section{Discussion}

Instead of the common single trials, neural responses to sequences of three aversive images during ER tasks were examined. Participants were instructed to apply AD or CR strategies based on the instruction. We focused on activation of amygdala as the key neural underpinning of the generation and regulation of negative emotional experiences [22-30]. Previous research on emotion regulation indicated a differential activation of amygdala during different cognitive ER tasks [16]. Based on previous findings that have underlined the importance of functional connectivity within limbic-cortical circuitry during emotion regulation [31], we conducted amygdala-centered connectivity investigations to reveal temporally related brain regions.

Results of the whole-brain analysis provided evidence for a differential recruitment of specific control systems in $\mathrm{AD}$ and $\mathrm{CR}$, which is based on the distinguished characteristics of each strategy. In our paradigm, consistent with the previous findings about cognitive reappraisal of negative emotions [13, 15], CR increased activity in medial, dorsolateral, and ventrolateral PFC regions stronger than $\mathrm{AD}$. Involvement of this network suggests the cognitive effort during application of CR strategies. Furthermore, stronger activation of IPL in parietal lobe, which have been implicated in shifting attention [32], could be shown in AD. Overall, differential neural activation patterns for the two instructed strategies was consistent with the previous reports $[6,16]$, mainly based on different cognitive demands of the two strategies [33]. The findings about the differential neural responses is notable, because the outcome of regulating emotions has been implied to be different in attentional deployment and cognitive reappraisal [34]. In our study, it may be taken as indirect evidence that participants followed the instructions.

The ROI-analysis proved that the time course of amygdala activation was distinctive in CR and AD. Previous investigations of amygdala's response to single presentations of negative images have shown modulation of amygdala activity within the first $2 \mathrm{~s}$ that participants attempted to reappraise a negative image, followed by a peak in about 1-2 $\mathrm{s}$ and a subsequent normalization [35]. By application of a unique study design, which involved multiple negative pictures, we could observe that the fMRI signal in amygdala showed an increase in course of ER and it rose stronger during CR than during $\mathrm{AD}$ tasks. An increasing involvement of amygdala in CR emphasizes that the distinction between emotion appraisal and control systems are not absolute.

Emotion regulatory processes in CR can activate further encoding of emotional stimuli through attention, expectations and memories [36, 37], which is neutrally represented in the enhanced amygdala activation. The significant difference of amygdala activation level between the two tasks is compatible with the previous findings [38], which showed 
that the time course of neural responding is differential between subtypes of cognitive strategies. More specifically, a reduced amygdala activation has been shown in distraction as compared to reappraisal $[6,16,17]$. Based on the earlier neuroimaging findings $[39,40]$, it is quite acknowledged that attentional shift affects the sensory encoding of emotional stimuli in the limbic system and the amygdala and reduces the response of these emotion-sensitive regions.

Meta-analyses of neuroimaging data have suggested a greater involvement of frontal cortical regions in the modulation of amygdala activity when subjects engage cognitive strategies of ER $[32,41]$. For further exploration, we extended the findings of the amygdala ROI-analysis by computing the correlations of amygdala activities with other cortical regions of the brain and compared these connectivity measures between $\mathrm{CR}$ and $\mathrm{AD}$ strategies. Our results confirmed that amygdala and cortical regions within left PFC were anticorrelated in CR, suggesting a higher negative connectivity in fronto-amygdala circuits during reappraisal task. We propose that this connectivity pattern is essential to emotion regulation efforts based on CR. Although AD and CR are both considered to be antecedent-focused forms of ER and pose cognitive demands [42], reappraisal of a negative visual scene involves inhibition of the initial negative interpretation, constructing a more positive one and bringing the alternative appraisals to the working memory [43]. Therefore, reappraisal may uniquely drive the down-regulating connections between working memory, executive control, and semantic structures in prefrontal cortex and amygdala.

We presented a sequence of aversive pictures to induce negative emotions, thereby during $\mathrm{CR}$ a new reappraisal strategy was required for each picture. The activation of amygdala ascended during late phase of CR task as successive negative images were demonstrated, and for each image a new reappraisal strategy was needed. This is a remarkable finding, since the cognitive reappraisal of negative emotions is thought to down-regulate the amygdala activation. For further investigations based on this result, we decided to examine the changes of the amygdala connectivity with time. In a post hoc analysis, we compared the measures of amygdala connectivity between the EARLY and LATE periods of ongoing CR blocks. The results of the connectivity analyses provided evidence for an altered amygdala connectivity in later periods of ongoing CR. In fact, the negative connectivity to frontal regions appeared to be reduced during the late periods of CR effort, which may account for the late escalation of the amygdala activation during reappraisal of aversive stimuli. Our paradigm implied that the CR strategies must have been repeatedly adjusted and a new reappraisal strategy was required for each picture, which might have increased the load of cognitive control during the CR blocks. Interpretation of the BOLD-derived functional connectivity is generally very challenging and in our analysis the direction of the information flow is still an unanswered question. Based on the proposed anatomical connections and emotion regulation models from the existing literature [15, 41], we suggest that the repetitive adjustments of regulation strategies may represent a high cognitive demand which interferes with the antecedent-focused cognitive control of emotion.

By involving blocks of multiple aversive images in our paradigm we aimed at capturing the changes in the local activation and connectivity of amygdala. In fact, we could obtain data that suggest the inverse relationship between PFC and the amygdala is compromised when sequences of aversive images elicit negative emotions and the ER strategy requires a high demand for mental flexibility. Emotion regulation contains indices of processes that influence generation, experience and expression of emotions [44], which implies an interactive dynamics in the neural structures that subserve ER. In particular, cognitive reappraisal is known to have its impact early in the emotion appraisal process [45]. All together, we suggest that emotion-related processes in the brain may be overlapping, which is necessary to allow integrating new information as soon as it is available.

In line with previous studies that have highlighted a regulatory PFC-amygdala interaction, our result revealed an inverse relationship between lateral and medial PFC and amygdala emotion regulation by $\mathrm{CR}$ strategies. The anatomical pathways, which are mediating this interaction are considered to be dependent on intrinsic anatomical connectivity and circuitries involving ACC and OFC, given the fact that lateral $\mathrm{PFC}$ receives only few direct inputs from amygdala [46]. Understanding functional and anatomical interactions between amygdala and regulatory prefrontal regions is important, because its deficits have been described across major psychiatric disorders [47-50]. The therapeutic relevance of these pathways has been emphasized by studies reporting that symptom reduction following behavioral and pharmacological interventions is accompanied by normalization of deficient amygdala-prefrontal coupling [51].

\section{Conclusion}

The current study advances our understanding of the interaction of regulatory cognitive regions and amygdala by showing that cognitive reappraisal of a sequence of aversive scenes is associated with a subsequent increase in amygdala activity. This observation highlights the importance of incorporating dynamic temporal features of emotion experience and related neural activations and interactions in emotion regulation models. Such findings at the neural level suggest that implementation of reappraisal strategies may influence experiential and behavioral indices of emotion over time. Particularly, AD strategies may serve as effective ER 
techniques in specific scenarios. Understanding of ongoing mechanisms of emotion regulation is essential for the mental health research. We suggest an advantage for the application of experiences, which are close to real-life situations, such as a sequence of related images or video sequences. However, special attention should be paid to install precise control conditions.

\section{Limitations}

We acknowledge some of the limitations of the present study. First, the restriction to negative emotions might restrain the generality of the findings. Second, no behavioral measurements were conducted as an outcome of voluntarily regulation. We did not collect self-evaluations of emotion, because the use of explicit self-report of emotional experience bears the risk of introducing biases of action and, possibly, reward anticipation. Third, it is possible that the two strategies have their maximal effects in different parts of the amygdala. However, our imaging parameters do not permit precise localization of activations within amygdala sub-nuclei and so it is not possible for the present data to address this differentiation in amygdala activations. Last, our data underwent two different analysis methods (GLMbased and connectivity-based), using different fMRI analysis platforms based on specific requirements of each analysis. Although we do not expect notable effects on the reliability of the reported results, we acknowledge that the use of one single analysis platform should be clearly preferred.

Acknowledgements We would like to acknowledge the support of the BrainGain Program of the Netherlands Ministry of Economic Affairs and the Netherlands Ministry of Education, Culture and Science. KM is supported by the German Research Foundation (DFG; IRTG 2150, MA2631/4 - 1) and the German Ministry for Education and Research (BMBF; APIC: 01EE1405A, 01EE1405B, 02EE1405C).

\section{Compliance with ethical standards}

Conflict of interest The authors declare that they have no conflict of interest.

\section{References}

1. Gross JJ, John OP (2003) Individual differences in two emotion regulation processes: implications for affect, relationships, and well-being. J Pers Soc Psychol 85:348-362

2. Berking M, Wupperman P (2012) Emotion regulation and mental health: recent findings, current challenges, and future directions. Curr Opin Psychiatry 25:128-134. https://doi.org/10.1097/ YCO.0b013e3283503669

3. Berking M, Wupperman P, Reichardt A et al (2008) Emotionregulation skills as a treatment target in psychotherapy. Behav Res Ther 46:1230-1237. https://doi.org/10.1016/j.brat.2008.08.005
4. Fassbinder E, Schweiger U, Martius D et al (2016) Emotion regulation in schema therapy and dialectical behavior therapy. Front Psychol 7:1373. https://doi.org/10.3389/fpsyg.2016.01373

5. Ray RD, McRae K, Ochsner KN, Gross JJ (2010) Cognitive reappraisal of negative affect: converging evidence from EMG and self-report. Emotion 10:587-592. https://doi.org/10.1037/ a0019015

6. Kanske P, Heissler J, Schönfelder S et al (2011) How to regulate emotion? neural networks for reappraisal and distraction. Cereb Cortex 21:1379-1388. https://doi.org/10.1093/cercor/bhq216

7. Hermann A, Kress L, Stark R (2016) Neural correlates of immediate and prolonged effects of cognitive reappraisal and distraction on emotional experience. Brain Imaging Behav. https://doi. org/10.1007/s11682-016-9603-9

8. Allard ES, Kensinger EA (2017) Cognitive emotion regulation in adulthood and old age: positive gaze preferences across two strategies. Aging Neuropsychol Cogn. https://doi. org/10.1080/13825585.2017.1279265

9. Ochsner KN, Gross JJ (2008) Cognitive emotion regulation: insights from social cognitive and affective neuroscience. Curr Dir Psychol Sci 17:153-158. https://doi.org/10.111 1/j.1467-8721.2008.00566.x

10. Ochsner KN, Silvers JA, Buhle JT (2012) Functional imaging studies of emotion regulation: a synthetic review and evolving model of the cognitive control of emotion. Ann N Y Acad Sci 1251:E1-E24. https://doi.org/10.1111/j.1749-6632.2012.06751 . $\mathrm{x}$

11. Zilverstand A, Parvaz MA, Goldstein RZ (2017) Neuroimaging cognitive reappraisal in clinical populations to define neural targets for enhancing emotion regulation. A systematic review. Neuroimage 151:105-116. https://doi.org/10.1016/j.neuroimage .2016.06.009

12. Kalisch R (2009) The functional neuroanatomy of reappraisal: time matters. Neurosci Biobehav Rev 33:1215-1226. https://doi. org/10.1016/j.neubiorev.2009.06.003

13. Buhle JT, Silvers JA, Wager TD et al (2014) Cognitive reappraisal of emotion: a meta-analysis of human neuroimaging studies. Cereb Cortex 24:2981-2990. https://doi.org/10.1093/cercor/ bht 154

14. Morawetz C, Bode S, Derntl B, Heekeren HR (2017) The effect of strategies, goals and stimulus material on the neural mechanisms of emotion regulation: a meta-analysis of fMRI studies. Neurosci Biobehav Rev 72:111-128. https://doi.org/10.1016/j. neubiorev.2016.11.014

15. Kohn N, Eickhoff SB, Scheller M et al (2014) Neural network of cognitive emotion regulation - an ALE meta-analysis and MACM analysis. Neuroimage 87:345-355. https://doi.org/10.1016/j.neuro image.2013.11.001

16. McRae K, Hughes B, Chopra S et al (2010) The neural bases of distraction and reappraisal. J Cogn Neurosci 22:248-262. https:// doi.org/10.1162/jocn.2009.21243

17. Dörfel D, Lamke J-P, Hummel F et al (2014) Common and differential neural networks of emotion regulation by detachment, reinterpretation, distraction, and expressive suppression: a comparative fMRI investigation. Neuroimage 101:298-309. https:// doi.org/10.1016/j.neuroimage.2014.06.051

18. Lang PJ, Bradley MM, Cuthbert BN (2008) International affective picture system (IAPS): affective ratings of pictures and instruction manual. Technical Report A-8. UNiversity of Florida, Gainsville, FL

19. Eickhoff SB, Stephan KE, Mohlberg H et al (2005) A new SPM toolbox for combining probabilistic cytoarchitectonic maps and functional imaging data. Neuroimage 25:1325-1335. https://doi. org/10.1016/j.neuroimage.2004.12.034

20. Bzdok D, Laird AR, Zilles K et al (2013) An investigation of the structural, connectional, and functional subspecialization in the 
human amygdala. Hum Brain Mapp 34:3247-3266. https://doi. org/10.1002/hbm. 22138

21. Whitfield-Gabrieli S, Nieto-Castanon A (2012) Conn: a functional connectivity toolbox for correlated and anticorrelated brain networks. Brain Connect 2:125-141. https://doi.org/10.1089/brain .2012 .0073

22. Zald DH (2003) The human amygdala and the emotional evaluation of sensory stimuli. Brain Res Brain Res Rev 41:88-123

23. Phillips ML, Drevets WC, Rauch SL, Lane R (2003) Neurobiology of emotion perception I: the neural basis of normal emotion perception. Biol Psychiatry 54:504-514

24. Murphy FC, Nimmo-Smith I, Lawrence AD (2003) Functional neuroanatomy of emotions: a meta-analysis. Cogn Affect Behav Neurosci 3:207-233

25. Phan KL, Wager T, Taylor SF, Liberzon I (2002) Functional neuroanatomy of emotion: a meta-analysis of emotion activation studies in PET and fMRI. Neuroimage 16:331-348. https://doi. org/10.1006/nimg.2002.1087

26. Dolan RJ, Vuilleumier P (2003) Amygdala automaticity in emotional processing. Ann N Y Acad Sci 985:348-355

27. Adolphs R, Spezio M (2006) Role of the amygdala in processing visual social stimuli. Prog Brain Res 156:363-378

28. Gallagher M, Chiba AA (1996) The amygdala and emotion. Curr Opin Neurobiol 6:221-227

29. Phelps EA, LeDoux JE (2005) Contributions of the amygdala to emotion processing: from animal models to human behavior. Neuron 48:175-187. https://doi.org/10.1016/j.neuron.2005.09.025

30. Davis M, Whalen PJ (2001) The amygdala: vigilance and emotion. Mol Psychiatry 6:13-34

31. Banks SJ, Eddy KT, Angstadt M et al (2007) Amygdala-frontal connectivity during emotion regulation. Soc Cogn Affect Neurosci 2:303-312. https://doi.org/10.1093/scan/nsm029

32. Ochsner K, Gross J (2005) The cognitive control of emotion. Trends Cogn Sci 9:242-249. https://doi.org/10.1016/j. tics.2005.03.010

33. Strauss GP, Ossenfort KL, Whearty KM (2016) Reappraisal and distraction emotion regulation strategies are associated with distinct patterns of visual attention and differing levels of cognitive demand. PLoS One 11:e0162290. https://doi.org/10.1371/journ al.pone. 0162290

34. Bebko GM, Franconeri SL, Ochsner KN, Chiao JY (2011) Look before you regulate: Differential perceptual strategies underlying expressive suppression and cognitive reappraisal. Emotion 11:732-742. https://doi.org/10.1037/a0024009

35. Ochsner KN, Ray RD, Cooper JC et al (2004) For better or for worse: neural systems supporting the cognitive down- and upregulation of negative emotion. Neuroimage 23:483-499. https:// doi.org/10.1016/j.neuroimage.2004.06.030

36. Vuilleumier P (2005) How brains beware: neural mechanisms of emotional attention. Trends Cogn Sci 9:585-594. https://doi. org/10.1016/j.tics.2005.10.011
37. Phelps EA (2006) Emotion and cognition: insights from studies of the human amygdala. Annu Rev Psychol 57:27-53. https://doi. org/10.1146/annurev.psych.56.091103.070234

38. Schönfelder S, Kanske P, Heissler J, Wessa M (2014) Time course of emotion-related responding during distraction and reappraisal. Soc Cogn Affect Neurosci 9:1310-1319. https://doi.org/10.1093/ scan/nst116

39. Liberzon I, Taylor SF, Fig LM et al (2000) Limbic activation and psychophysiologic responses to aversive visual stimuli interaction with cognitive task. Neuropsychopharmacology 23:508-516. https ://doi.org/10.1016/S0893-133X(00)00157-3

40. Hariri AR, Bookheimer SY, Mazziotta JC (2000) Modulating emotional responses: effects of a neocortical network on the limbic system. Neuroreport 11:43-48

41. Quirk GJ, Beer JS (2006) Prefrontal involvement in the regulation of emotion: convergence of rat and human studies. Curr Opin Neurobiol 16:723-727. https://doi.org/10.1016/j.conb.2006.07.004

42. Gross JJ (2007) Handbook of emotion regulation. Guilford Press, New York

43. Messina I, Bianco S, Sambin M, Viviani R (2015) Executive and semantic processes in reappraisal of negative stimuli: insights from a meta-analysis of neuroimaging studies. Front Psychol 6:956. https://doi.org/10.3389/fpsyg.2015.00956

44. Gross JJ (1998) The emerging field of emotion regulation: an integrative review. Publ Found 2:271-299

45. Goldin PR, McRae K, Ramel W, Gross JJ (2008) The neural bases of emotion regulation: reappraisal and suppression of negative emotion. Biol Psychiatry 63:577-586. https://doi.org/10.1016/j. biopsych.2007.05.031

46. Amaral DG, Price JL (1984) Amygdalo-cortical projections in the monkey (Macaca fascicularis). J Comp Neurol 230:465-496. https ://doi.org/10.1002/cne.902300402

47. New AS, Hazlett EA, Buchsbaum MS et al (2007) Amygdalaprefrontal disconnection in borderline personality disorder. Neuropsychopharmacology 32:1629-1640. https://doi.org/10.1038/ sj.npp. 1301283

48. Wolf RC, Herringa RJ (2016) Prefrontal-amygdala dysregulation to threat in pediatric posttraumatic stress disorder. Neuropsychopharmacology 41:822-831. https://doi.org/10.1038/npp.2015.209

49. Johnstone T, van Reekum CM, Urry HL et al (2007) Failure to regulate: counterproductive recruitment of top-down prefrontalsubcortical circuitry in major depression. J Neurosci 27:88778884. https://doi.org/10.1523/JNEUROSCI.2063-07.2007

50. Lindner P, Flodin P, Larm P et al (2018) Amygdala-orbitofrontal structural and functional connectivity in females with anxiety disorders, with and without a history of conduct disorder. Sci Rep 8:1101. https://doi.org/10.1038/s41598-018-19569-7

51. Sheline YI, Barch DM, Donnelly JM et al (2001) Increased amygdala response to masked emotional faces in depressed subjects resolves with antidepressant treatment: an fMRI study. Biol Psychiatry 50:651-658 